\title{
Spatial Resolution Improvement by Merging MERIS-ETM Images for Coastal Water Monitoring
}

\author{
Audrey Minghelli-Roman, Laurent Polidori, Sandrine Mathieu-Blanc, Lionel Loubersac, and François Cauneau
}

\begin{abstract}
The Medium Resolution Imaging Spectrometer (MERIS) was launched in March 2002 and has been providing images since June 2002. Before its launch, we had implemented a method to improve its resolution by merging its images with Landsat Enhanced Thematic Mapper images in order to preserve the best characteristics of the two images (spatial, spectral, temporal). We now present the results of this method for real MeRIS images (level $1 \mathrm{~b}$ and 2 ) in a coastal area. The robustness of the method is studied as well as the influence of the delay between the acquisitions of the two images .
\end{abstract}

Index Terms - Fusion, Landsat Enhanced Thematic Mapper (ETM), Medium Resolution Imaging Spectrometer (MERIS).

\section{INTRODUCTION}

\section{$\mathrm{O}$} CEAN colour monitoring is usually based on optical remote sensing with spatial resolutions around $1 \mathrm{~km}$. This spatial scale is available with such sensors as the National Oceanic and Atmospheric Adminitration's Avanced Very High Resolution Radiometer, the Polarization and Directionality of the Earth'Reflectances Instrument, or Seaviewing Wide Field-of-view Sensor, which cover one to several thousand kilometers in a single swath and providing very short revisit periods. The Medium Resolution Imaging Spectrometer (MeRIS) sensor, launched on board ENVISAT in 2002, was designed for sea colour observation, with a $300-$ meter spatial resolution, 15 programmable spectral bands and a 3 day revisit period. Tree hundred meter is a high resolution for an oceanographic sensors, but it is still too rough for coastal water monitoring, where physical and biological phenomena require better spatial resolution [1]. On the opposite, multispectral Landsat Enhanced Thematic Mapper (ETM) images offer a suitable spatial resolution, but have only 4 spectral bands in the visible and near infrared spectrum, allowing poor spectral characterization. A few years ago, in order to combine the spectral resolution of MeRIS and the spatial resolution of Landsat ETM, we had implemented a merging method proposed by Zhukov et al. [2]. Before the launch of ENVISAT, we applied this method to simulated MeRIS images [3].

Manuscript received May 18 ; revised August 30, 2005.

A. Minghelli-Roman is with the Universite de Bourgogne, LE2I, UMR-CNRS 5158, 21078 DIJON Cedex, France (email : audrey.roman@u-bourogne.fr).

L. Polidori is with IRD, Laboratoire Régional de Télédétection, 97323 Cayenne Cedex, French Guyana

S. Mathieu-Blanc is with Alcatel Alenia Space Industries, 06156 Cannes la Bocca Cedex, France

L. Loubersac is with IFREMER, DEL-DRV LER/LR, 34203 Sète Cedex, France

F. Cauneau is with Ecole des Mines de Paris, Centre d'Energétique, BP207, 06904 Sophia-Antipolis Cedex, France

Digital Object Identifier 10.1109/LGRS.2005.861699
This method is now applied to real MeRIS images. Two product levels are considered. Level $1 \mathrm{~b}$ contains radiance measurements at the top of the atmosphere for the calibrated and geocoded fifteen (15) MeRIS bands. Level 2 contains normalized surface reflectance and several geophysical and biophysical parameters such as algal pigment index, suspended sediment, Rayleigh-corrected Vegetation Indices, aerosol type, cloud albedo. The method was tested for radiance (level $1 \mathrm{~b}$ ) and reflectance (level 2) over a coastal area of approximately $30 \times 30 \mathrm{~km}^{2}$ located around the Thau lagoon (southern France).

The main steps of the method are briefly recalled and the results are presented for levels $1 \mathrm{~b}$ and 2 . A validation method is proposed based on a statistical quality criterion, namely, the ERGAS parameter. In our previous paper we had suggested that the delay between the two images could have an impact on the fusion relevance in case of landscape evolution. This constraint is analysed as well. Eventually, the potential and limitations of this resolution improvement approach are discussed.

\section{METHOD}

ETM images have high spatial resolution and low spectral resolution compared to MeRIS images. A MeRIS-ETM merging method was presented in detail by Minghelli-Roman et al. [3]. First of all, the MeRIS image is geometrically coregistered with the ETM image. Then, a multispectral classification is applied to the ETM image in order to divide its pixels into $\mathrm{N}_{\mathrm{c}}$ classes. The only request is that all pixels must belong to a class (i.e no pixel remains unclassified).

Each MeRIS pixel covers 100 pixels of the ETM classification. The proportion of each class is computed within each MeRIS pixel. For each class, a mean spectral profile is obtained by solving an algebraic system. The last step consists of substituting each classified pixel with its corresponding spectral profile. This output image is a $30 \mathrm{~m}$ spatial resolution (ETM spatial resolution) and 15 spectral bands (MeRIS spectral resolution).

For MeRIS level 2 images, we noticed that "black pixels" were located on land-water borders [Fig. 5 (a)-(c)]. Since atmospheric corrections use different methods on land and water, these mixed pixels are not corrected and their MeRIS level 2 reflectance is set to zero for all spectral bands. These pixels are used neither to solve the algebraic system nor to determine the spectral profile of the class. In order not to leave black pixels in the resulting image, we replace the classified ETM pixels by the spectral profile obtained for the pixels belonging to the same class. 


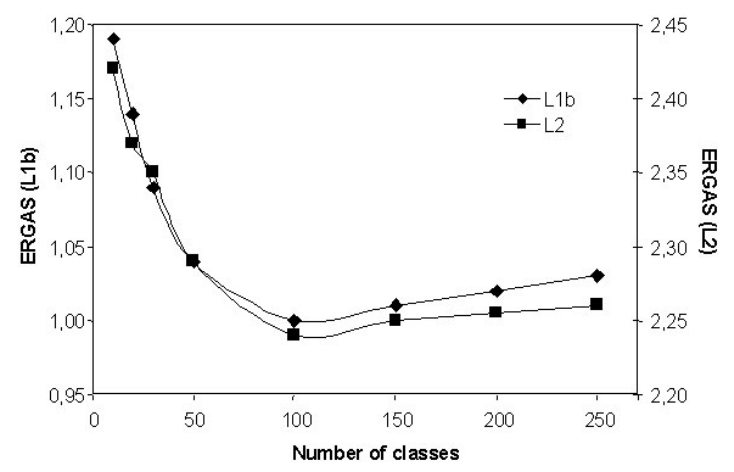

Fig. 1. ERGAS versus number of classes. The optimal number of classes is given by the minimum value of the ERGAS parameter, i.e. 100 for level $1 \mathrm{~b}$ and 2 images.

\section{OPTIMISATION OF THE NUMBER OF CLASSES}

\section{AND VALIDATION}

For all images, the number of classes has been optimised. Classifications have been run on the ETM image with different numbers of classes in order to assess the influence of this number on the fusion output.

The ERGAS parameter, based on an RMSE estimation [4], is chosen as a robustness criterion (1). This statistical parameter is often used for evaluation of fusion techniques [5]. It compares the absolute radiometric values between MeRIS original image and the one resulting from the fusion. Resolution of pixels resulting from the fusion is degraded to $300 \mathrm{~m}$ to be compared to the original MeRIS ones. This subsampling was performed by pixel averaging, thus considering the MeRIS Modulation Transfer Function (MTF) as perfect for the resolution decrease. This approximation has statistically no effect on the comparison.

$$
\operatorname{ERGAS}(\mathrm{X}, \mathrm{Y})=100 * \frac{h}{l} \sqrt{\frac{1}{\mathrm{nb}} \sum_{\lambda=1}^{\mathrm{nb}} \frac{\left[\operatorname{RMSE}(X, Y, \lambda]^{2}\right.}{[\operatorname{mean}(X-Y, \lambda)]^{2}}}
$$

with

$$
\begin{aligned}
& \mathrm{X} \text { original MeRIS image, } \\
& \mathrm{Y} \text { resulting image of fusion } \\
& \mathrm{h} \text { the ETM resolution }(30 \mathrm{~m}), \\
& 1 \text { the MeRIS resolution }(300 \mathrm{~m}) \\
& \lambda \text { the spectral band, } \\
& \mathrm{nb} \text { the total number of spectral bands. }
\end{aligned}
$$

The ERGAS is affected by two main factors. The first one is the classification applied to the ETM image. It reduces the radiometric variability. The number of classes has no intrinsic physical significance. It is only a consequence of the radiometric variability within the TM image.

The second factor is the delay between the two image acquisitions. It increases the probability of landscape changes. If the classification applied to ETM is merged with a MeRIS image acquired a long time later, the fusion may become inconsistent.

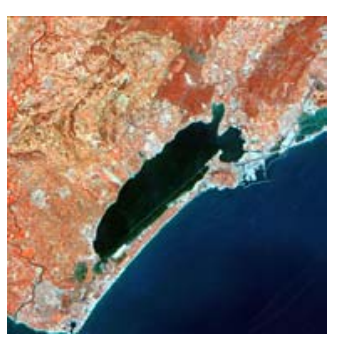

(a)

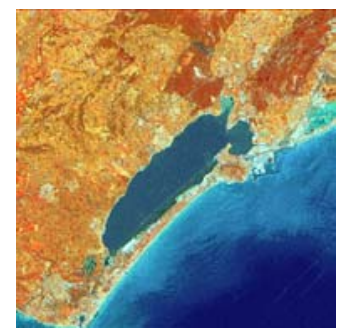

(c)

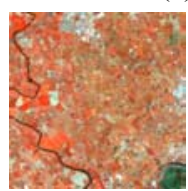

(d)

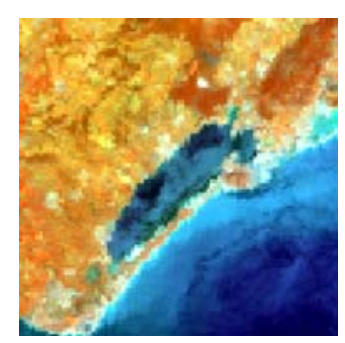

(b)

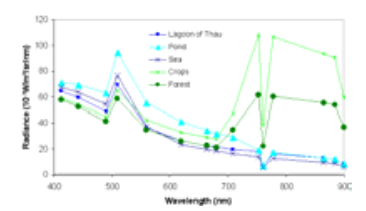

(d)

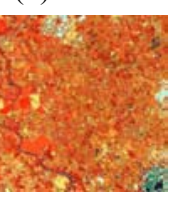

(f)
Fig. 2. (a) ETM Colour Composite (30m, 6 spectral bands), (b) MeRIS (Level 1b) Colour Composite $(300 \mathrm{~m}, 15$ spectral bands) acquired on August 14, 2002, (c) Fusion result (30m, 15 spectral bands), (d) Spectral radiance profiles of some items, (e) - (g).

\section{RESULTS}

\section{A. Results on MeRIS level $1 \mathrm{~b}$ images}

Fig. 1 shows that the optimal number of classes is around 100 with an ERGAS parameter equal to 1.00. Fig. 2(a) shows a colour composite of the input ETM image (1000x1000 pixels) and Fig. 2(b) the input MeRIS image (100x100 pixels) in radiance. A zoom factor of 10 has been applied to this image in order to emphasise the difference between ETM and MeRIS resolutions. Eventually, the resulting image [Fig. 2(c)] is characterized by 15 spectral bands and a $30 \mathrm{~m}$ resolution. A spatial improvement is visible when comparing fig. 2(b) and 2(c).

MeRIS image spectra are well preserved, as suggested by the colour image, as well as the geometric resolution of the ETM image. The output image has the spatial characteristics of ETM and the spectral and radiometric characteristics of MeRIS (radiance image). The spectral profiles in radiance of several pixels containing different items are presented: Thau lagoon, pond, sea, crops and forest [Fig. 2(d)]. The location of these items, which is not possible on the input MeRIS image because of its coarse resolution, is much easier in the improved image. Indeed, the fusion method has drastically improved the spatial resolution without losing MeRIS spectral quality. For each item, spectral profiles in radiance can therefore be extracted from output image $(30 \mathrm{~m}, 15$ spectral bands).

It is observed that the boundaries between classes are noticeable especially on areas with reduced radiometric dynamics, e.g. over the stretch of sea water. 
To show how the method behaves on the borders of region (rivers, road, fields), a small area has been enlarged [Fig. 2(e)(g)]. One can notice that fields and road borders are better preserved than river borders. This is due first to the high ratio of resolution between the two images equal to 10 then to the high radiometry difference between water and soil within MeRIS mixed pixels:

\section{B. Results on MeRIS Level 2 images}

The same fusion has been applied to the same MeRIS image but on the level 2 product. The optimal number of classes is also 100 , and the ERGAS parameter is then equal to 2.24 . This difference in ERGAS parameter values is mainly due to the artefact that MeRIS level 2 images exhibit some "black pixels" located on land-water borders.

Comparing the number of classes with the simulated case (equal to 150 [3]), we remark that different radiometric and geomorphologic conditions provide different optimizations.

\section{TEMPORAL CONSTRAINS DUE TO LANDSCAPE EVOLUTION}

The revisit period of an imaging sensor depends on satellite orbit and on viewing geometry (off-nadir angle and swath width). The revisit periods of MeRIS and ETM being different and not synchronized, fusion can generally not be applied to images acquired simultaneously. Because ETM Landsat has a long revisit period (image acquisitions can occur every 16 days at minimum, and much more in cloudy conditions) and MeRIS has a wide swath ( images can be acquired more frequently i.e. every 3 days at minimum), the fusion of ETM and MeRIS can then become inconsistent if the landscape has changed between the 2 acquisitions.

For example, heliosynchronous orbits are not synchronized with tide cycles, so that two images have no reason for having the same tide elevation. This may be a severe limitation in coastal areas. Similarly, seasonal landscape changes appear if the time delay exceeds a few months. The robustness of the fusion may then be evaluated with the increasing delay between ETM and MeRIS images acquisition.

\section{A. Results on MeRIS level $1 \mathrm{~b}$ images}

The same ETM image $(1000 * 1000$ pixels $)$ acquired on August 16, 2002 has been merged with 3 different MeRIS level $1 \mathrm{~b}$ images acquired on August 14, 2002, October 13, 2002 and May 3, 2003. The delays between ETM and MeRIS images are respectively 2 days, 2 months and 9 months. All MeRIS images have been geometrically co-registered with the ETM image. Fig. 3 (a)-(c) shows these 3 MeRIS images $(100 * 100$ pixels $)$

A radiometric evolution can be observed between MeRIS images due to seasonal changes. The first image has been acquired in summer, the second one in autumn and the last one in spring. We can note that inland pixels have brighter tones in the second image than in the third one, because of the higher photosynthesis activity in spring. It is also noticeable that the lagoons and some of the ponds have a pale green hue in autumn and summer and a dark green hue in spring. This can be explained by a higher eutrophication in summer and autumn than in spring.

The assumption of the geometric stability between the 2 acquisitions has been made. If necessary this assumption will be verified during the validation part, by using the ERGAS parameter. Fig. 3 (d)-(f) shows the visual results of these fusions.

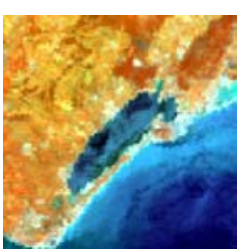

$14 / 08 / 02$

(a)

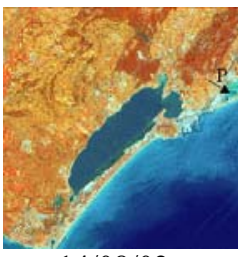

$14 / 08 / 02$

(d)

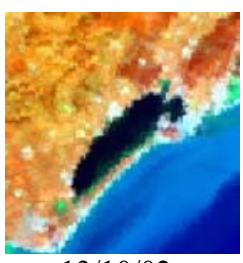

$13 / 10 / 02$

(b)

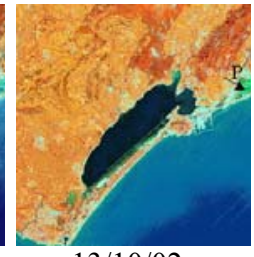

$13 / 10 / 02$

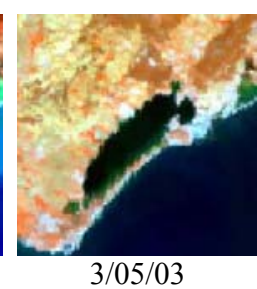

(c)

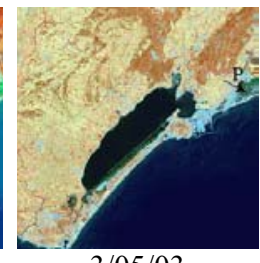

$3 / 05 / 03$

(f)
Fig. 3. MeRIS (Level 1b) colour composite images. (a) August 14, 2002, (b) October 13, 2002, (c) May 3, 2003. Fusion results (with MeRIS level 1b). (d) August 14, 2002, (e) October 13, 2002, (f) May 3,2003

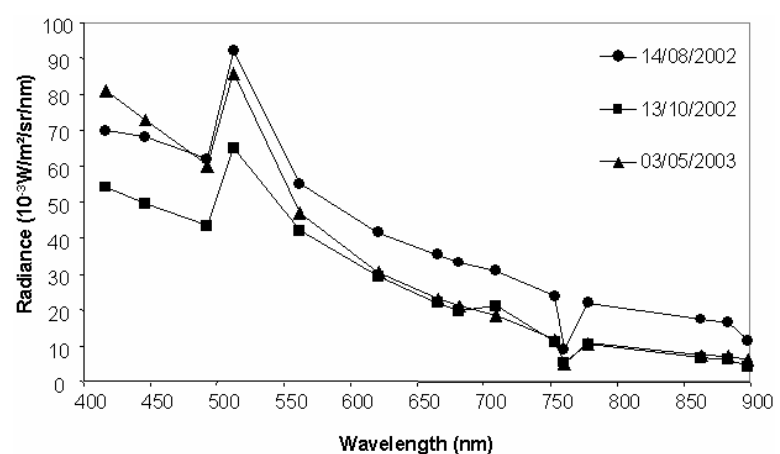

Fig. 4. Temporal spectral profile of the pixel indicated as P in Fig. 3 (in radiance)

A visual comparison shows that the seasonal landscape evolutions are preserved. With the fusion, the spectral evolution of a landscape object can be tracked in the ETM image. Fig. 4 shows the spectral evolution of the pixel indicated as point $\mathrm{P}$, located at the middle of a pond, at the East of Thau lagoon.

Fig. 4 shows a decrease of the chlorophyll peak between August 14, 2002 and October 13, 2002 and an increase between October 13, 2002 and May 3, 2003. This evolution is explained by a strong eutrophication of some lagoons which receive fluvial waters containing organic matter and chemical fertilizers from cultivated fields situated upstream in watersheds. This eutrophication increases in summer under specific atmospheric conditions decreases in autumn and winter and increases again in spring.

\section{B. Results on MeRIS level 2 images}

The same ETM image (1000*1000 pixels) acquired on the August 16, 2002 has been merged with 3 different MeRIS Level 2 images acquired on September 9, 2003, August 22, 2003 and September 13, 2003. The image acquisition dates are different from level 1 images. The objective is not to compare the results between level 1 and level 2 images (the results would have been similar) but to show that the fusion is possible between two image levels according to the required product. The delays between ETM and MeRIS image 


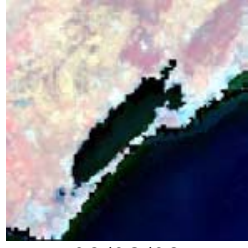

09/08/03

(a)

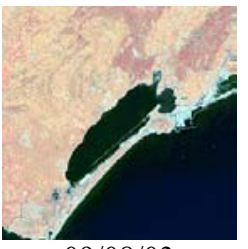

09/08/03

(d)

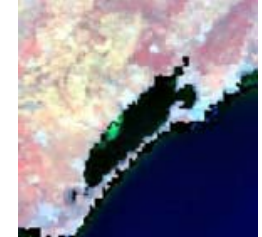

$22 / 08 / 03$

(b)

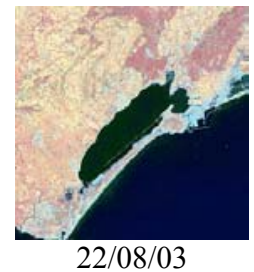

(e)

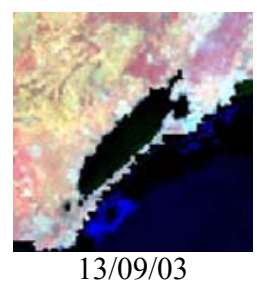

(c)

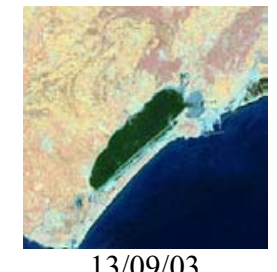

(f)
Fig. 5. MeRIS (Level 2) colour composite images. (a) September 9, 2003, (b) August 22, 2003, (c) September 13, 2003. Fusion results (with MeRIS level 2). (d) August 9, 2003, (e) August 22, 2003, (f) September 3, 2003

acquisitions are respectively 358, 371 days and 393 days. MeRIS images have been geometrically co-registered on the ETM image. Fig. 5 (a)-( c) shows these 3 extracts of MeRIS images $(100 * 100$ pixels).

The pale colours of MeRIS images are due to atmospheric corrections which use different methods on land and water. The black pixel artefact for level $1 \mathrm{~b}$ images is noticed on landwater borders [Fig. 5(a)-(c)]. The image of the September 13, 2003 has many pale blue pixels which are the result of inaccurate land-water masking.

The August 22, 2003 image shows a bright spot on the Thau lagoon, produced by an anoxic crisis. This crisis was due to the eutrophication and was resulting from specific meteorological and environmental conditions.

Fig. 5 (d)-(f) shows the fusion results for the 3 dates. One can notice that MeRIS radiometric values are globally preserved on water and land. Black pixels have also disappeared and have been replaced by reflectance values computed from other pixels of the same class located somewhere else in the image as explained in the method description. It is particularly noticeable on Fig. 5(f) where the fusion has removed a important black pixel artefact.

A typical temporal effect can be seen in Fig. 5(e), where the white spot on the Thau lagoon disappeared. It is due to its absence in the original ETM image. The high reflectance of this spot has then been "diluted" in the other classes covering the Thau lagoon. This phenomenon confirms that this fusion method is only valid for a landscape with limited changes between the acquisition dates of ETM and MeRIS. Indeed, the spectral profile of a landscape object can be monitored only if it is present in the original ETM image.

Results of the ERGAS parameter calculation are given in Table I and Table II.

Table I shows that the ERGAS parameter increases with the delay between images acquisitions. This confirms the temporal limitation of our fusion method. Ranchin et al. [4] have empirically fixed a fusion validity limit for ERGAS $=3$. The first three fusions with MeRIS level $1 \mathrm{~b}$ images are valid because the landscape has not geometrically changed between the two dates.
TABLE I

MERIS LEVEL 1: DATE ACQUISITION, DELAY, BETWEEN ETM AND MERIS ACQUISITION, ERGAS PARAMETER

\begin{tabular}{|c|c|c|c|}
\hline Image & Date & $\begin{array}{c}\text { Delay between ETM and } \\
\text { MeRIS acquisitions } \\
\text { (days) }\end{array}$ & ERGAS \\
\hline TM & $16 / 08 / 02$ & & 1.05 \\
\hline \multirow{2}{*}{$\begin{array}{c}\text { MeRIS } \\
\text { level 1 }\end{array}$} & $14 / 08 / 02$ & -2 & 1.33 \\
\cline { 2 - 4 } & $13 / 10 / 02$ & 58 & 1.92 \\
\hline
\end{tabular}

TABLE II

MERIS LEVEL 2: DATE ACQUISITION, DELAY, BETWEEN ETM AND MERIS ACQUISITION, ERGAS PARAMETER

\begin{tabular}{|c|c|c|c|}
\hline Image & Date & $\begin{array}{c}\text { Delay between ETM and } \\
\text { MeRIS acquisitions } \\
\text { (days) }\end{array}$ & ERGAS \\
\hline TM & $16 / 08 / 02$ & 358 & 3.88 \\
\hline \multirow{2}{*}{$\begin{array}{c}\text { MeRIS } \\
\text { level 2 }\end{array}$} & $09 / 08 / 03$ & 371 & 2.86 \\
\cline { 2 - 4 } & $22 / 08 / 03$ & 393 & 4.66 \\
\cline { 2 - 4 }
\end{tabular}

The fusion with MeRIS level 2 images is more critical as revealed by the ERGAS parameter which exceeds or is close to 3. Two reasons explain these high values, first the important delay between the two images acquisitions minimum 358 days, second the apparition of the white spot on the Thau lagoon which brought a geometrical change into the landscape. However, the ERGAS values given in Table 1 and 2 cannot be compared by absolute values because level 2 images are not obtained by a linear transformation from level 1b images. The "black pixels" artefact affects the ERGAS parameter with a bias of 1.23 (Fig. 1), for example, for the image of the August 14, 2002.

\section{DISCUSSION}

The method proposed for MeRIS spatial resolution improvement has several advantages. Classical unmixing methods [6]- [8] require an a priori knowledge of endmembers and their spectral profiles. This knowledge requires minimum ground information and becomes meaningless if the image does not contain any pure pixel. For such methods, the number of end-members cannot exceed the number of spectral bands in order to make the system invertible and therefore to ensure that one solution exists. For the chosen fusion method, the number of end-members is only bounded by the number of available pixels in the low resolution image.

Concerning preprocessing requirements, classical methods handle absolute spectra so that radiometric preprocessing is needed such as absolute calibration and atmospheric, directional and topographic corrections, which are not required for this method.

Beyond these operational advantages, the most interesting potential of this method is the effective resolution improvement due to the fusion with a higher resolution image, while other methods only provide a proportion of each endmember inside each pixel without any subpixel location information.

However, some limitations need to be considered, some of which have already been identified [3]. For example, first the merged image is only an approximation of the pseudo image 
acquired by a virtual $30 \mathrm{~m}$ resolution and 15 spectral bands instrument, and the radiometry of pixels belonging to the same class corresponds to the average radiometry measured by this virtual instrument. Second, if a landscape object was not present in the ETM image due to a drastic landscape change, it will not be present in the merged image. On the contrary, a global seasonal evolution, in which the pixels belonging to the same class have similar changes, has less effect on the fusion result because it does not modify the initial classification. In both cases, the landscape evolution is more likely to occur when time delay increases between ETM and MeRIS image acquisitions, and its effects on the fusion relevance can be evaluated by comparing the ERGAS values for different time delays.

\section{CONCLUSION}

This letter has shown how a MeRIS image can be merged with an ETM image in order to synthesize a new product with the best characteristics of each sensor, namely, the spatial resolution of ETM, and the spectral resolution and the frequent revisiting of MeRIS. The method, which had been tested on simulated MeRIS images before the launch of ENVISAT, was applied to real images over a coastal area. The effects of landscape evolution, which tend to increase as the time delay between the two acquisitions becomes longer, were analysed using the ERGAS parameter as a robustness criterion. The advantages and limitations of this resolution improvement method have been discussed. This experiment confirms the potential of this method for coastal water monitoring. However, a further validation should be carried out in areas where drastic changes use to occur, like in tropical coastal zones.

\section{REFERENCES}

[1] A. Minghelli, “Apport et perspectives de l'imagerie hyperspectrale pour la télédétection des paysages natures et Agricoles", PhD. thesis, Nice Sophia-Antipolis University, 1999.

[2] B. Zhukov, D. Oertel, F. Lanzl, and G. Reinhäckel, "Unmixing-based multi sensor multi-resolution image fusion", IEEE Trans. Geosci. Remote Sensing, vol. 37, pp. 1212-1226, 1999.

[3] A. Minghelli-Roman., M. Mangolini M., M. Petit and L. Polidori, "Spatial Resolution Improvement of MeRIS Images by Fusion with TM images", IEEE Trans. Geosc. Remote Sensing, vol. 39, pp. 1533-1536, 2001

[4] T. Ranchin, B. Aiazzi, L. Alparone, S. Baronti and L. Wald, "Image fusion - the ARSIS concept and some successful implementation schemes", ISPRS J. Photogram. Remote Sensing, vol. 58, pp. 4-18, 2003

[5] T. Wehrmann, R.R. Colditz, M. Bachmann, K. Steinnocher, S. Dech, "Evaluation of image Fusion techniques", Remote Sensing and GIS for Environmental studies, Vol. 113, pp. 296-302, 2005

[6] Y.H. Hu, H.B. Lee, and F.L. Scapace, "Optimal linear spectral unmixing", IEEE Trans. Geosci. Remote Sensing, vol. 37, pp. 639-644, 1999.

[7] F. Van Der Meer, "Iterative spectral unmixing (ISU)", Int. J. Remote Sensing, vol. 20, pp. 3431-3436, 1999.

[8] J.J. Settel and N.A. Drake, "Linear mixing and the estimation of ground cover proportions", Int. J. Remote Sensing, vol. 14, pp. 1159-1177, 1993. 